Research Article

\title{
Network Pharmacology-Based Systematic Analysis of Molecular Mechanisms of Dingji Fumai Decoction for Ventricular Arrhythmia
}

\author{
Yi Liang $\mathbb{D}^{1},{ }^{1}$ Bo Liang $\mathbb{D},{ }^{2}$ Xin-Rui Wu $\mathbb{D}^{1},{ }^{1}$ Wen Chen $\mathbb{D}^{1},{ }^{1}$ and Li-Zhi Zhao $\mathbb{D}^{3}$ \\ ${ }^{1}$ Southwest Medical University, Luzhou, China \\ ${ }^{2}$ Nanjing University of Chinese Medicine, Nanjing, China \\ ${ }^{3}$ Hospital (T.C.M.) Affiliated to Southwest Medical University, Luzhou, China
}

Correspondence should be addressed to Li-Zhi Zhao; zhaolizhi@swmu.edu.cn

Received 31 January 2021; Revised 31 March 2021; Accepted 28 April 2021; Published 10 May 2021

Academic Editor: Guoqi Zhu

Copyright $(92021$ Yi Liang et al. This is an open access article distributed under the Creative Commons Attribution License, which permits unrestricted use, distribution, and reproduction in any medium, provided the original work is properly cited.

Background. Dingji Fumai Decoction (DFD), a traditional herbal mixture, has been widely used to ventricular arrhythmia (VA) in clinical practice in China. However, research on the bioactive components and underlying mechanisms of DFD in VA is still scarce. Methods. Components of DFD were collected from TCMSP, ETCM, and literature. The chemical structures of each component were obtained from PubChem. Next, SwissADME and SwissTargetPrediction were applied for compounds screening and targets prediction of DFD; meanwhile, targets of VA were collected from DrugBank and Online Mendelian Inheritance in Man (OMIM). Then, the H-C-T-D network and the protein-protein interaction (PPI) network were constructed based on the data obtained above. CytoNCA was utilized to filter hub genes and VarElect was used to analyze the relationship between genes and diseases. At last, Metascape was employed for systematic analysis on the potential targets of herbals against VA, and AutoDock was applied for molecular docking to verify the results. Results. A total of 434 components were collected, 168 of which were qualified, and there were 28 shared targets between DFD and VA. Three function modules of DFD were found from the PPI network. Further systematic analysis of shared genes and function modules explained the potential mechanism of DFD in the treatment of VA; molecular docking has verified the interactions. Conclusions. DFD could be employed for VA through mechanisms, including complex interactions between related components and targets, as predicted by network pharmacology and molecular docking. This work confirmed that DFD could apply to the treatment of VA and promoted the explanation of DFD for $\mathrm{VA}$ in the molecular mechanisms.

\section{Introduction}

In recent years, cardiovascular diseases are the leading cause of death in China [1]. All cardiac conditions, especially ischemic heart disease, can lead to arrhythmias [2]. Among all arrhythmias, ventricular arrhythmia (VA) has the highest mortality. VA is a common but life-threatening disease, mainly including ventricular premature contraction, ventricular tachycardia, ventricular flutter, and ventricular fibrillation, with a clinical presentation that ranges from no symptoms to cardiac arrest [2]. VA is usually generated by all-caused enhanced automaticity or abnormal automaticity, myocardial ischemia, delayed afterdepolarizations, and structural heart disease with reentry [3-5]. To prevent adverse events of VA, millions of patients were treated with beta-receptor-blockers, INa antagonists, IKr antagonists, nondihydropyridine calcium antagonists, and other drugs suggested by the 2017 American Heart Association/American College of Cardiology/Heart Rhythm Society guideline [2], but the control of VA is still far from ideal.

Traditional Chinese medicine (TCM) has a clinicalbased development history of over 2000 years $[6,7]$. Some researchers mentioned that TCM could be used to treat a variety of diseases, including VA [8-10]. Besides, related studies suggested that, compared with Western medicine only, patients suffering from various diseases can benefit 
more from a TCM and Western medicine combined therapy strategy [11-22]. Moreover, several TCM, such as Shensong Yangxin Capsule [9] and Wenxin Keli [23], have been transformed into commercial products for the treatment of cardiovascular-related diseases. Such convenient approaches greatly promoted the development of TCM worldwide.

Dingji Fumai Decoction (DFD), consisting of Chuanxiong Rhizoma (Chuanxiong), Jujubae Fructus (Dazao), Poria cocos (Schw.) Wolf (Fuling), Cinnamomi Ramulus (Guizhi), Silktree Albizia Bark (Hehuanpi), Osdraconis (Fossiliaossiamastodi) (Longgu), Ostrea Gigas Thunberg (Muli), Ziziphi Spinosae Semen (Suanzaoren), Radix Polygalae (Yuanzhi), and Licorice (Gancao), is widely used for VA and obtains encouraging results [24], and previous experiments revealed that one of the underlying mechanisms is class I antiarrhythmic property [25]. To make DFD more recognized, it is essential to ensure the efficacy and safety of DFD. Since multiple components are contained in DFD, it can generate interactions on multiple targets. Based on the theory of network pharmacology, we constructed network relationships between "component-target pathways" to explore the mechanisms of drugs or herbs [26]. Here, we analyzed the mechanisms of DFD in the treatment of VA systematically (Figure 1). At first, we obtained its components and potential targets against VA. Then, the protein-protein interaction (PPI) of potential targets against VA was constructed. Next, a systematic analysis of potential targets and biofunctional modules was conducted. Meanwhile, the interactions between the components of DFD and key targets were confirmed using molecular docking.

\section{Methods}

2.1. Chemical Structures Construction. The Traditional Chinese Medicine Systems Pharmacology database and analysis platform (TCMSP) [27] and the Encyclopedia of Traditional Chinese Medicine (ETCM) [28] are web-based herb databases, providing comprehensive and standardized information for the commonly used herbs. In this study, the components of each herb in DFD were obtained from TCMSP, ETCM, and published literature. To make the components recognizable for the subsequent analysis work, after removing duplicates, the structure of each component was collected from PubChem [29].

\subsection{Gastrointestinal Absorption (GA) and Drug-Likeness (DL)} Prediction. Increasingly researchers found that TCM despite their impressive in vitro findings demonstrate less or negligible in vivo activity, resulting in poor absorption and hence poor bioavailability [30]. The absorption, distribution, metabolism, and excretion (ADME) of the drug must be considered by the researcher and developer [31]. Bioabsorption is highly multifactorial but is primarily driven by GA [32]. Besides, DL assesses qualitatively the chance for a molecule to be an oral drug with respect to bioavailability [31]. It was constructed that the estimation of ADME before the drug development studies reduces the possibility of failure [33]. In the mechanism explaining DFD, GA and DL were evaluated using SwissADME, a free tool that could evaluate DL, GA, pharmacokinetics, and medicinal chemistry friendliness of small molecules [31]. After uploading the structure of each compound to SwissADME, if the prediction results of the component suggested high possibility of both GA and DL, it met our inclusion criteria and was adopted for the next screening [31].

2.3. Target Prediction and Verification. In the treatment of diseases, not all absorbable components work; therefore, we filtered out the components with bioactive components from all absorbable components using SwissTargetPrediction, an online tool that can evaluate compounds with a score by fitting a multiple logistic regression on various subsets of known actives to weight structure similarity parameters [34]. Here, we uploaded the structure of each component to SwissTargetPrediction to predict potential targets of DFD, and all possible targets were adopted.

Online Mendelian Inheritance in Man (OMIM) is a knowledge base providing the latest information of human genes [35], and DrugBank is a freely available and comprehensive web resource providing drug-target and drug interaction information [36]. Taking "ventricular arrhythmia" or "arrhythmia of ventricular origin" as a keyword, we obtained VA-related targets from OMIM and DrugBank. Taking the intersection of DFD and VA targets, the common targets between DFD and VA were considered the therapeutic targets of DFD against VA, as described previously [37].

PPI is one of the cores of cellular processing. The analysis of PPI makes the interactions of proteins clear and helps to explain the function of possible protein complexes or functional modules [38]. STRING is a web database providing online analysis of PPI [38]. After uploading the common targets to STRING, we constructed the PPI network. Then, the result was imported to Cytoscape (version 3.8.0) for further analysis [39]. CytoNCA plugin in Cytoscape was applied to analyze centrality of certain targets and evaluate protein interaction networks [40].

The VarElect online tool can analyze direct and indirect links between genes and diseases [41]. In this study, the link relationships of potential targets of DFD against VA were analyzed with VarElect; the results helped determine which targets will be included in the next molecular docking.

2.4. Biology Functional Analysis. Since Gene Ontology (GO) and Kyoto Encyclopedia of Genes and Genomes (KEGG) can contribute to the interpretation of system-level data and enable new discoveries [42], in this study, Metascape was employed for GO and KEGG analysis to further explore the complex mechanism of DFD in the treatment of VA. Metascape is a web-based platform providing gene annotation, functional enrichment, and interactome analysis services; monthly database update could keep our analysis results up to date [42]. In our work, GO and KEGG terms with $P<0.01$ were considered significantly enrichment analyses.

2.5. Molecular Docking. Molecular docking was used to assess interactions between components and hub targets; the 4 hub 

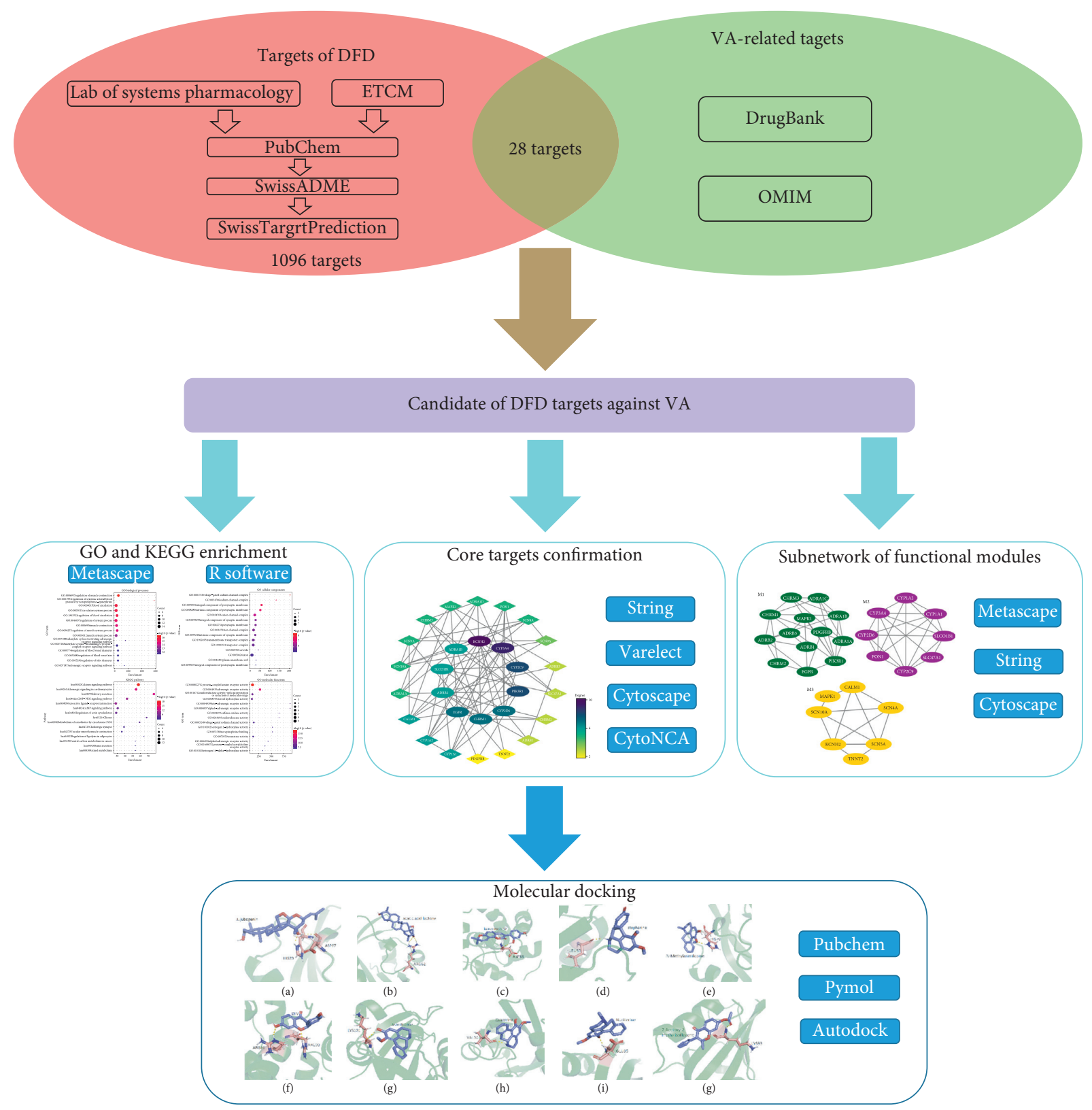

Figure 1: Flowchart of this work.

targets connected to VA closely were included. The structures of these targets were collected from Protein Data Bank [43]. AutoDock and PyMOL were employed for molecular docking, PyMOL was used to remove the water molecules and isolate proteins of the moleculars [44]. AutoDock was used to add the hydrogen and calculate Gasteiger charges of the moleculars and add the hydrogen for the ligands [45]. At last, molecular docking was conducted using AutoDock to assess the binding energy; the lower the binding energy, the more stable the docking modules [45].

\section{Results}

3.1. Chemical Structures Construction. After searching TCMSP, ETCM, and literature [27, 28], a total of 434 compounds were collected, including 92, 73, 76, 38, 19, 70,
12, 36, and 18 compounds in Licorice, Chuanxiong Rhizoma, Jujubae Fructus, Poria cocos (Schw.) Wolf, Cinnamomi Ramulus, in Silktree Albizia Bark, Ostrea Gigas Thunberg, Ziziphi Spinosae Semen, and Radix Polygalae. Later, the structure of each component was collected from PubChem.

3.2. GA and DL Prediction. The structures were uploaded to SwissADME; after screening the GA and DL and removing duplicates, 168 components qualified, including 80, 20, 27, 11, 4, 19, 4, 10, and 2 in Licorice, Chuanxiong Rhizoma, Jujubae Fructus, Poria cocos (Schw.) Wolf, Cinnamomi Ramulus, Silktree Albizia Bark, Ostrea Gigas Thunberg, Ziziphi Spinosae Semen, and Radix Polygalae, respectively. Interestingly, several qualified components are owned by more than one herbal; more information about the 
prediction results was placed in the Supplementary Materials. All qualified components were adopted for the next screening.

3.3. Target Prediction and Verification. After removal of duplicates, a total of 1096 potential targets of DFD were collected from SwissTargetPrediction. Meanwhile, a total of 260 known therapeutic targets for VA were obtained from OMIM and DrugBank. Taking the intersection of DFD and VA targets, there were 28 shared targets; based on the data obtained above, the Herb-Compound-Targets-Disease ( $\mathrm{H}$ C-T-D) network was constructed. The H-C-T-D network was composed of 147 nodes (DFD, VA, 7 herbals, 110 bioactive compounds, and 28 common targets) and 465 edges (Figure 2).

In further analysis, all 28 common targets were uploaded to STRING to construct the PPI network; the results were imported to Cytoscape to calculate the degree value of each gene using CytoNCA plugin and reconstruct the PPI network according to the degree value (Figure 3). There were three possible biofunctional modules divided from the PPI network (Figure 4). After screening by CytoNCA, the top 10 targets were defined as hub targets; the designations and topological parameters of hub targets are shown in Table 1.

We analyzed the 28 common targets using VarElect to investigate the correlation between targets and VA, and the results suggest that 23 targets were related to VA directly, whereas 5 targets were related to VA indirectly (Table 2); among these targets, Potassium Voltage-Gated Channel Subfamily H Member 2 (KCNH2), Sodium Voltage-Gated Channel Alpha Subunit 5 (SCN5A), Troponin T2-Cardiac Type (TNNT2), and Calmodulin 1 (CALM1) have the highest score of correlation.

3.4. Biology Functional Analysis. The enrichment analysis of GO and KEGG of the 28 common targets was analyzed using Metascape, the results were ranked by $-\log 10$ (P value), and the top 14 of each enrichment item are shown in Figure 5; besides, the functional analysis of the three potential biofunction modules divided from PPI network is shown in Table 3 .

In GO and KEGG enrichment analysis, terms with high enrichment scores suggest that the regulation of muscle contraction, regulation of systemic arterial blood pressure by norepinephrine-epinephrine, blood circulation, circulatory system process, adrenergic receptor activity, calcium signaling pathway, adenylate signaling in cardiomyocytes, cGMP-PKG signaling pathway, and neuroactive ligand-receptor interaction could be the most possible mechanisms of DFD in treating VA. For the 3 protein modules, Module 1 can regulate calcium signaling pathway, heart rate, and cAMP signaling pathway. Module 2 can regulate membrane depolarization during an action potential, striated muscle contraction, regulate adrenergic signaling in cardiomyocytes, and cation homeostasis. And Module 3 is more likely to possess the function of regulation of the metabolic process.
3.5. Molecular Docking. Molecular docking was conducted to calculate binding energy between components and hub targets, KCNH2 (PDB ID:1BYW), SCN5A (PDB ID: 4DCK), TNNT2 (PDB ID: 1J1D), and CALM1 (PDB ID: 1CDL); the 4 genes connected closely to VA were included in molecular docking. The docking information of components and hub targets was listed in Table 4, and the results suggested that components in DFD could interact with the hub targets against VA, following the principle that the lower the binding energy, the more stable the docking modules [45]. The lowest 10 binding energy docking modules are shown in Figure 6, the rest docking module could be found in the supplement, and all 10 components interacted with corresponding targets through hydrogen bond mainly.

The results show that Jujubogenin has the highest binding energy connected with HIS70 and ASP67 of KCNH2 (Figure 6(a)), acacic acid lactone connected with ARG62 of KCNH2 (Figure 6(b)), kanzonols W connected with ASP88 of TNNT2 (Figure 6(c)), stepharine connected with LEU86 of KCNH2 (Figure 6(d)), N-methylasimilobine connected with HIS70 of KCNH2 (Figure 6(e)), DFV connected with ARG86 and ARG90 of CALM1 (Figure 6(f)), asimilobine connected with LYS101 of KCNH2 (Figure 6(g)), cadaverine connected with VAL36 of KCNH2 (Figure 6(h)), nuciferine connected with GLU95 of KCNH2 (Figure 6(i)), and 7acetoxy-2-methylisoflavone connected with LYS93 of KCNH2 (Figure 6(j)).

\section{Discussion}

VA is a fatal disease, typical drugs may benefit patients, but its side effects such as respiratory diseases, liver and kidney damage, and bradyarrhythmia can never be ignored. Fortunately, long-time clinical work was told that DFD is an effective herb mixture against antiarrhythmia. Since its excellent clinical efficacy, we conducted a Real-World Trial that included more than 160 patients who suffered premature ventricular contractions to assess the safety and efficacy of DFD for VA and the results demonstrate that DFD combined with metoprolol has better efficacy and safety than placebo combined with metoprolol [24]. Besides, we explored the cellular electrophysiological mechanism of DFD with Chinese hamster ovary cells using whole-cell patch-clamp, and the result suggests that DFD indeed has antiarrhythmic effects based on its antioxidant potential, alleviation of $\mathrm{Na}^{+}-\mathrm{K}^{+}$-ATPase and connexin-43, and class I antiarrhythmic properties by suppressing $\mathrm{Nav}_{1.5}$ dose-dependently with an $\mathrm{IC}_{50}$ of $24.0 \pm 2.4 \mathrm{mg} / \mathrm{mL}$ [25]. In this study, the bioactive components and underlying molecular mechanisms of DFD in the treatment of VA were analyzed systematically.

Through related information collection and primary screening, we identified 28 potential targets of DFD in the treatment of VA. A PPI network was constructed with STRING and Cytoscape 3.8.0, the top $10^{\circ}$ value genes were selected as hub genes, and 3 function modules were divided based on their interactions. All potential genes were analyzed using VarElect, all 10 hub genes are suggested directly related to the treatment of VA, and among these genes, 


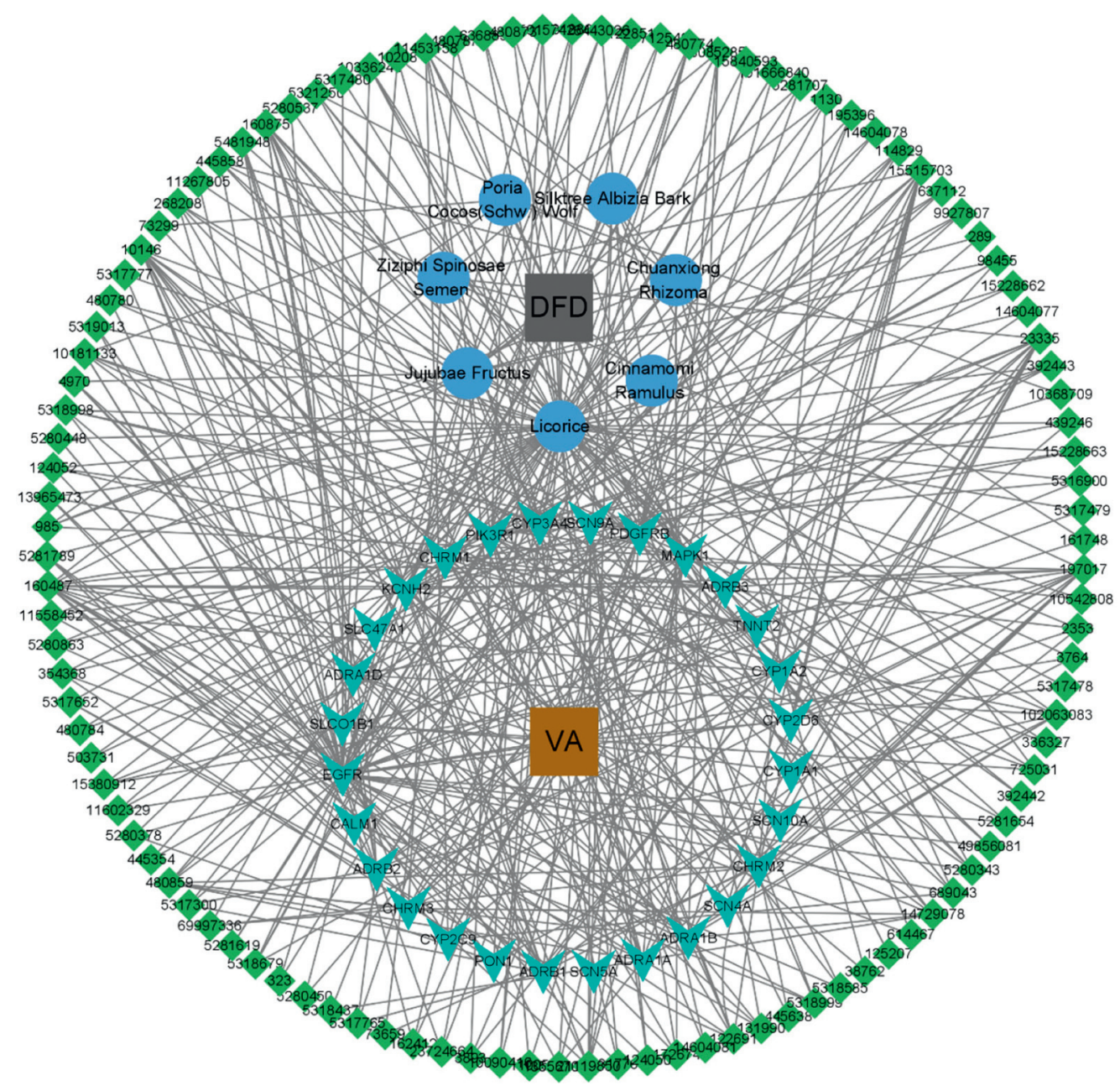

Figure 2: The H-C-T-D network of DFD. Grey and chocolate rectangles indicate DFD and VA. Blue round indicates the 7 herbal medicines comprising DFD. Green quadrilateral indicates the 110 active compounds and cyan shape V indicates the 28 shard targets, respectively.

KCNH2, TNNT2, and CALM1 as well as SCN5A have the highest scores of correlations; in other words, these 4 genes are the most promising targets for DFD against VA. Recently, KCNH2 could be a hot gene in the study of VA; it could mediate the rapidly activating component of the delayed rectifying potassium current in the heart. Previous research suggested that pathogenic variants in $\mathrm{KCNH} 2$ encoding may result in long QT syndrome [46]. Meanwhile, another research based on quantitative analysis of consortium disease cohorts and population controls pointed out that, among patients with long QT syndrome, the mutation probability of the $\mathrm{KCNH} 2$ gene is greater than $85 \%$ [47]. Besides, another research mentioned the coexpression of CACNA1C and KCNH2 reduces the arrhythmic events [48]. TNNT2 is another hub gene connected to arrhythmias; a genetic analysis suggested that TNNT2 was cosegregated in VAs and sudden death [49]. A study conducted using zebrafish embryos suggested that zebrafish embryos exposed to procymidone are more likely to alter transcription levels of TNNT2 and resulted in arrhythmia as well as increased heart rate finally [50]. Raffaele Coppini conducted a cohort study of patients with hypertrophic cardiomyopathy
(HCM), the outcome indicated that, among patients with HCM, most patients have a mutation in TNNT2, and these patients are more likely to suffer from arrhythmias and HCM in the future [51]. SCN5A is pivotal to cardiac electrical conduction and arrhythmic risk; a study provided a new effective therapy to reduce arrhythmia through downregulating the expression of SCN5A [52]. Similarly, there is a study that reported that a combination of quinidine/mexiletine reduces arrhythmia in patients with SCN5A gene mutation [53]. CALM1 is a regulator of voltage-dependent L-type calcium channels; its mutations are related to congenital arrhythmia [54]. Heterozygosity for the CALM1 mutation is causative of an arrhythmia syndrome [55]. Moreover, it can lead to catecholaminergic polymorphic ventricular tachycardia, idiopathic ventricular fibrillation, long QT syndrome, and even sudden death [56].

In the further, the results of GO and KEGG analysis elucidated that the regulation of systemic arterial blood pressure by norepinephrine-epinephrine, muscle contraction, blood circulation, circulatory system process, adrenergic receptor activity, calcium signaling pathway, adenylate signaling in cardiomyocytes, cGMP-PKG signaling pathway, 


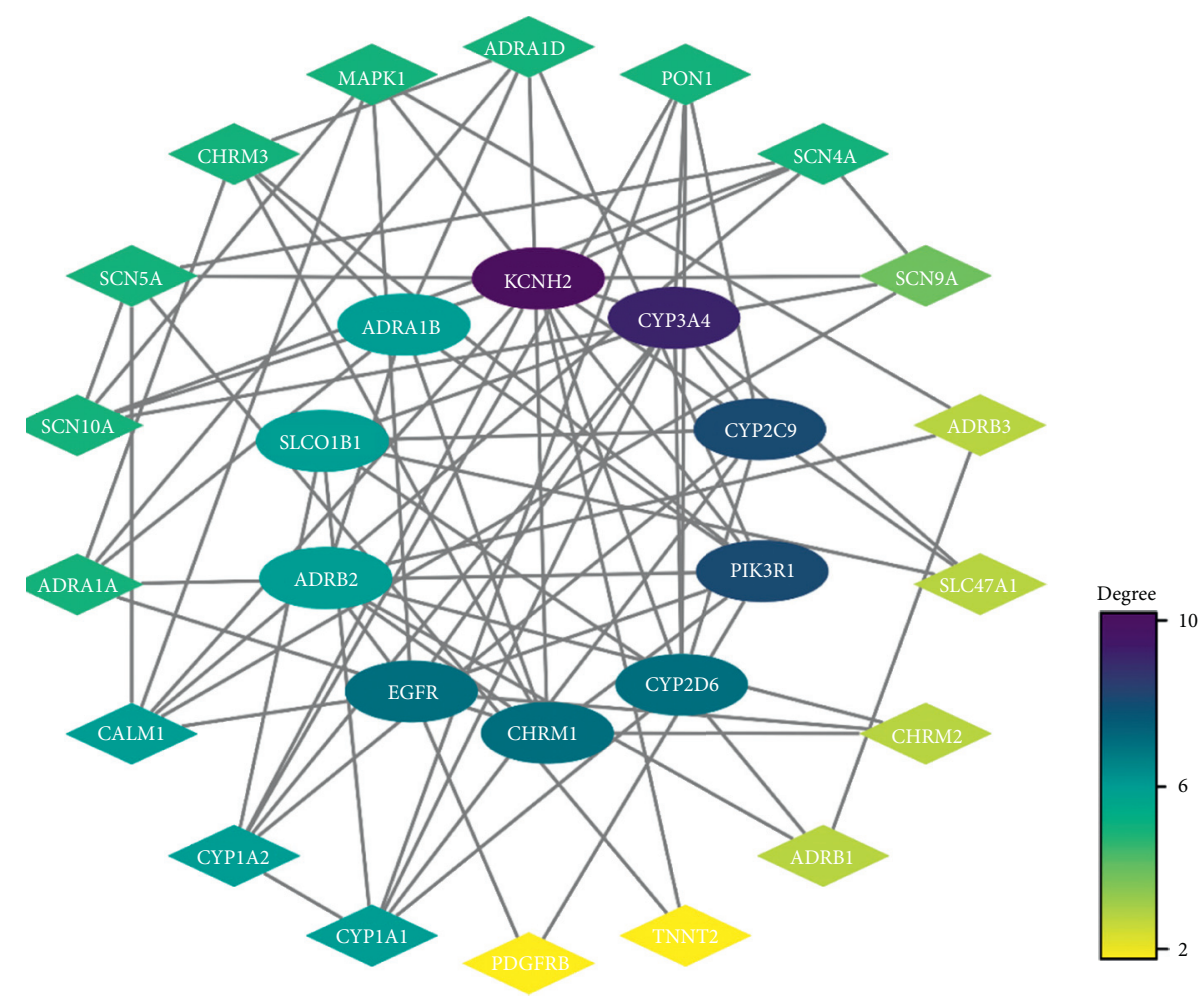

FIgURE 3: PPI network of potential targets of DFD against VA. Oval nodes on the inner circle indicate hub targets.

and neuroactive ligand-receptor interaction were highly enriched, which revealed the potential mechanisms of DFD in treating VA. Here, the regulation of the calcium signaling pathway is enriched by most of the hub genes. According to a study based on the genomic, transcriptomic, and proteomic data initiated by Dan E Arking, calcium signaling pathway plays an important role in both the depolarization and repolarization of myocardial ischemia; particularly in the repolarization, during the plateau phase of the cardiac action potential, prolonged inward $\mathrm{Ca} 2+$ currently leads to delays in ventricular myocyte repolarization [57]. Earlier research also mentioned that $\mathrm{Ca}^{2+}$ waves can form when the $\mathrm{Ca}^{2+}$ ion influx into the cell is increased, and the $\mathrm{Ca}^{2+}$ waves can generate depolarization that triggers arrhythmias [58], it is reasonable to speculate that the regulation of calcium signaling pathways of DFD may be one of the effective methods for VA. Reports also suggested that the adrenergic signaling can increase the transmural difference between $\mathrm{Ca}^{2+}$ ion transients duration and action potential duration, finally, promoting the formation of delayed afterdepolarizations, the regulation of adenylate cyclase-activating adrenergic receptor signaling pathway and adrenergic receptor signaling pathway of DFD for VA may antiarrhythmia in this way [59]. Adenylate cyclase-modulating G protein-coupled receptor signaling pathway can result in the regulation of $G$ protein-mediated signaling, which is of great importance for the regulation of heart rate and involved in arrhythmias [60]. Besides, as we mentioned above, the potential targets were divided into 3 function modules, as shown in Table 3. The enrichment analysis results indicate Module 1 can regulate calcium signaling pathway, heart rate, and cAMP signaling pathway, which suggest that Module 1 has great potential in the antiarrhythmia. Similarly, the results of enrichment analysis indicate that Module 2 were involved in the regulation of membrane depolarization during an action potential, striated muscle contraction, regulate adrenergic signaling in cardiomyocytes and cation homeostasis, pathways, which has illustrated the antiarrhythmic potential of Module 2. The enrichment analysis of Module 3 may not seem to be ideal, but we found that the 4 genes connected to VA closest are gathered in function Module 3, and it is reasonable to believe that Module 3 has antiarrhythmic effects. Furthermore, as is shown in Figure 5, the multiregulation in different aspects may benefit patients suffering from related diseases such as hypertension, cancer, and other diseases.

Nevertheless, the present study has some limitations. First, although we tried to find out all components, Osdraconis (Fossiliaossiamastodi) and Ostrea Gigas Thunberg, has only several pieces of research with several components, and were excluded for its poor possibility of GA and DL, but Osdraconis (Fossiliaossiamastodi) and Ostrea Gigas Thunberg played important roles in DFD; according to the theoretical system of TCM, both Osdraconis (Fossiliaossiamastodi) and Ostrea Gigas Thunberg can tranquilize the mind; further studies are needed to confirm the sedative mechanism of Osdraconis (Fossiliaossiamastodi) and Ostrea Gigas Thunberg. Moreover, DFD, as a traditional Chinese decoction, treats VA through multicomponent and multitarget, indicating that the underlying mechanisms are complex as described in our present study. Although we have made some identification on the mechanisms [25], 

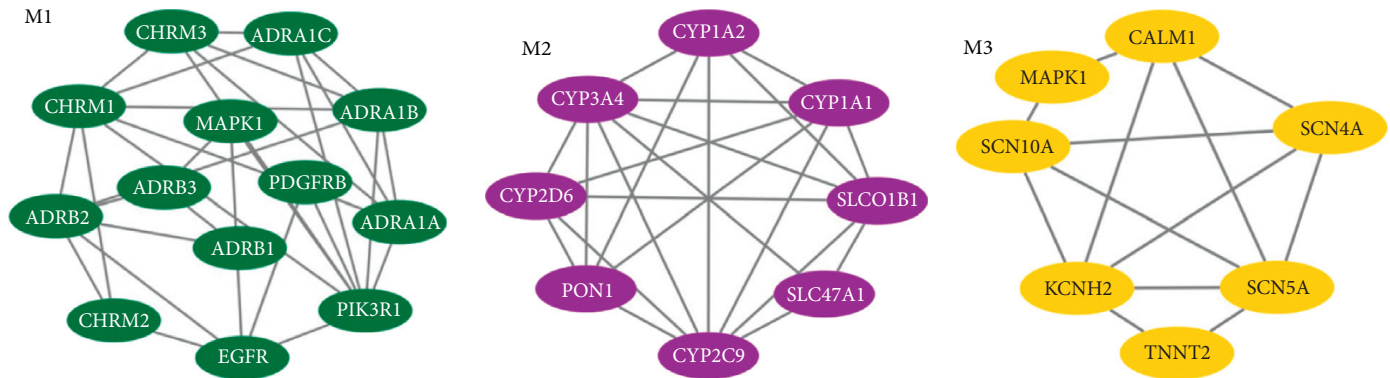

FIgURE 4: Three possible biofunctional modules divided from the PPI network.

TABLe 1: Designations and topological parameters of hub genes in the PPI network.

\begin{tabular}{lcccc}
\hline Gene symbol & Protein name & Degree & Betweenness centrality & Closeness centrality \\
\hline KCNH2 & Potassium voltage-gated channel subfamily H member 2 & 10 & 128.5 & 0.32 \\
CYP3A4 & Cytochrome P450 3A4 & 9 & 166.8 & 0.35 \\
CYP2C9 & Cytochrome P450 2C9 & 8 & 22.2 & 0.31 \\
PIK3R1 & Phosphatidylinositol 3-kinase regulatory subunit alpha & 8 & 146.7 & 0.31 \\
CHRM1 & Muscarinic acetylcholine receptor M1 & 7 & 79.2 & 0.27 \\
CYP2D6 & Cytochrome P450 2D6 & 7 & 59.1 & 0.32 \\
EGFR & Epidermal growth factor receptor & 6 & 228.3 & 0.36 \\
SLCO1B1 & Solute carrier organic anion transporter family member 1B1 & 3.9 & 0.28 \\
ADRA1B & Alpha-1B adrenergic receptor & 6 & 11.1 & 0.27 \\
ADRB2 & Beta-2 adrenergic receptor & 63.8 & 0.31 \\
\hline
\end{tabular}

TABLE 2: The connection information of shared genes.

\begin{tabular}{|c|c|c|c|}
\hline Genes & Description & Relationship & Score \\
\hline SCN5A & Sodium voltage-gated channel alpha subunit 5 & Directly & 445.8 \\
\hline $\mathrm{KCNH} 2$ & Potassium voltage-gated channel subfamily $\mathrm{H}$ member 2 & Directly & 409.76 \\
\hline TNNT2 & Troponin T2, cardiac type & Directly & 293.82 \\
\hline CALM1 & Calmodulin 1 & Directly & 222.12 \\
\hline SCN4A & Sodium voltage-gated channel alpha subunit 4 & Directly & 158.41 \\
\hline SCN10 A & Sodium voltage-gated channel alpha subunit 10 & Directly & 132.53 \\
\hline ADRB1 & Adrenoceptor beta 1 & Directly & 97.00 \\
\hline ADRB2 & Adrenoceptor beta 2 & Directly & 75.14 \\
\hline CYP2C9 & Cytochrome P450 family 2 subfamily $\mathrm{C}$ member 9 & Directly & 53.13 \\
\hline PDGFRB & Platelet-derived growth factor receptor beta & Directly & 46.86 \\
\hline CHRM2 & Cholinergic receptor muscarinic 2 & Directly & 46.86 \\
\hline MAPK1 & Mitogen-activated protein kinase 1 & Directly & 43.38 \\
\hline EGFR & Epidermal growth factor receptor & Directly & 43.38 \\
\hline ADRA1D & Adrenoceptor alpha 1D & Directly & 39.60 \\
\hline ADRB3 & Adrenoceptor beta 3 & Directly & 39.60 \\
\hline SCN9A & Sodium voltage-gated channel alpha subunit 9 & Directly & 35.42 \\
\hline CYP3A4 & Cytochrome P450 family 3 subfamily A member 4 & Directly & 30.68 \\
\hline CYP2D6 & Cytochrome P450 family 2 subfamily D member 6 & Directly & 25.05 \\
\hline PON1 & Paraoxonase 1 & Directly & 25.05 \\
\hline PIK3R1 & Phosphoinositide-3-kinase regulatory subunit 1 & Directly & 17.71 \\
\hline CYP1A1 & Cytochrome P450 family 1 subfamily A member 1 & Directly & 17.71 \\
\hline CHRM1 & Cholinergic receptor muscarinic 1 & Directly & 17.71 \\
\hline CYP1A2 & Cytochrome P450 family 1 subfamily A member 2 & Directly & 17.71 \\
\hline ADRA1A & Alpha-1A adrenergic receptor & Indirectly & 37.78 \\
\hline ADRA1B & Alpha-1B adrenergic receptor & Indirectly & 36.23 \\
\hline CHRM3 & Muscarinic acetylcholine receptor M3 & Indirectly & 28.09 \\
\hline SLCO1B1 & Solute carrier organic anion transporter family member $1 \mathrm{~B} 1$ & Indirectly & 13.50 \\
\hline SLC47A1 & Multidrug and toxin extrusion protein 1 & Indirectly & 11.33 \\
\hline
\end{tabular}

Notes: the score is an indication of the strength of the connection between the gene and the disease. 


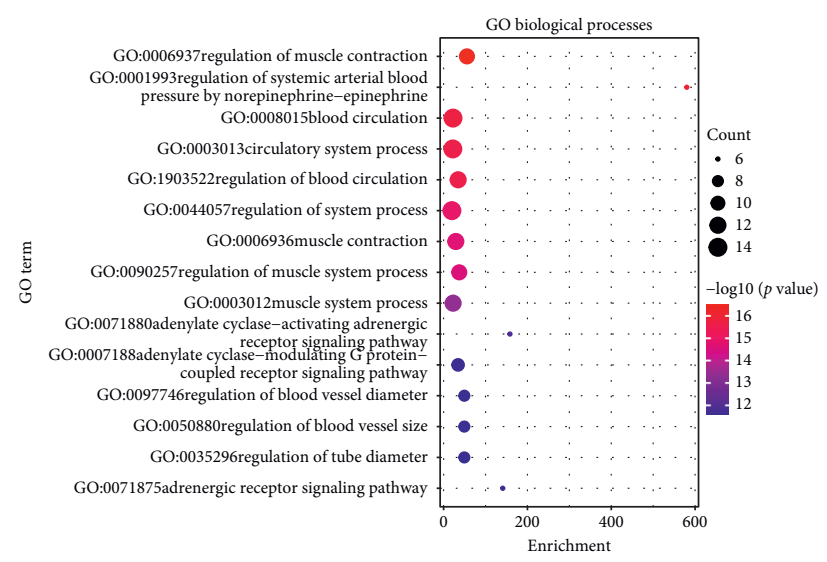

(a)

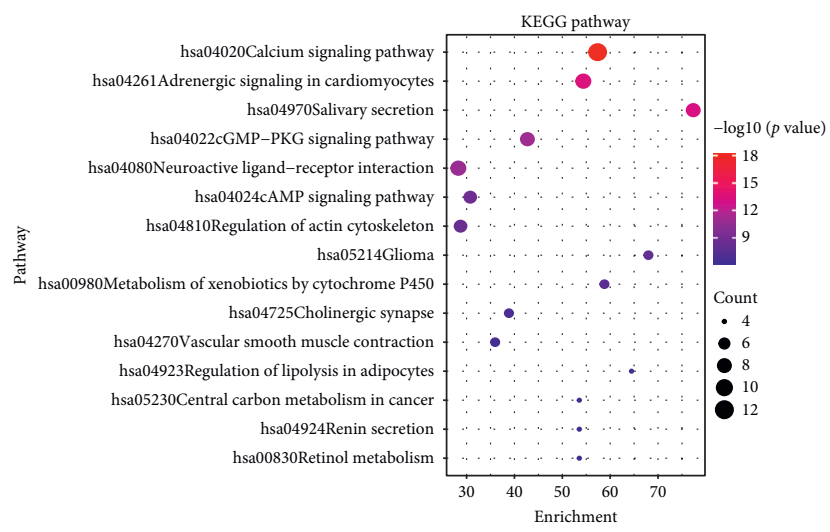

(c)

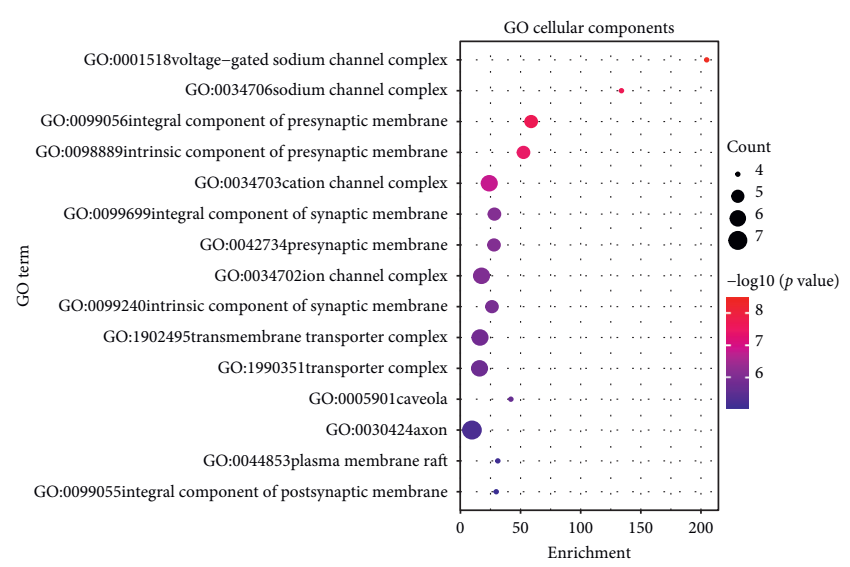

(b)

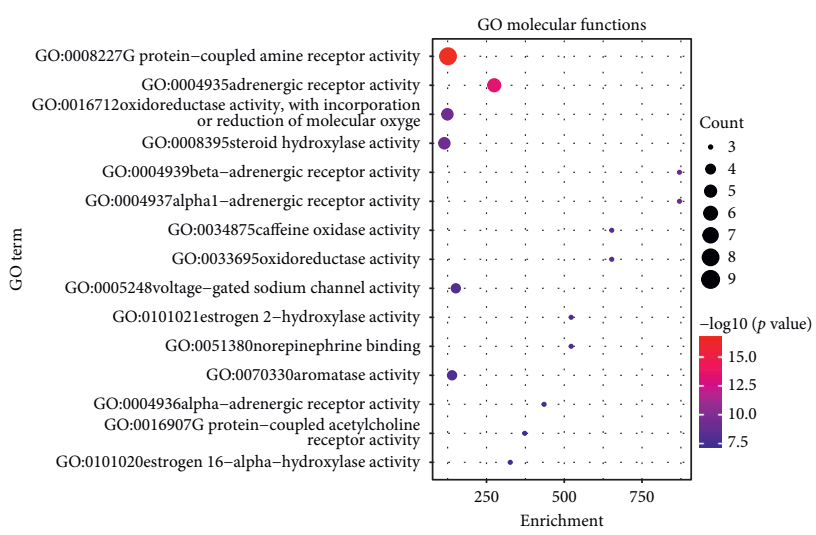

(d)

FIGURE 5: GO and KEGG enrichment analysis of potential targets.

TABLE 3: GO and KEGG enrichment analysis of the biofunction modules.

\begin{tabular}{lcc}
\hline Function modules & Description & Log $_{10}(P$ value $)$ \\
\hline & ko04020, calcium signaling pathway & -22.3027025 \\
Godule 1 & ko04810, regulation of actin cytoskeleton & -12.3777817 \\
& GO:0043410, positive regulation of MAPK cascade & -11.6704785 \\
& ko04024, cAMP signaling pathway & -10.5659921 \\
& GO:0086010, membrane depolarization during action potential & -9.71878217 \\
Module 2 & GO:0006941, striated muscle contraction & -12.7696584 \\
& ko04261, adrenergic signaling in cardiomyocytes & -11.8188992 \\
& GO:0019233, sensory perception of pain & -7.33482642 \\
GO:0055080, cation homeostasis & -5.53543107 \\
Module 3 & GO:0016098, monoterpenoid metabolic process & -3.03192747 \\
\hline & GO:0008202, steroid metabolic process & -13.3868655 \\
& GO:0006690, icosanoid metabolic process & -10.1366162 \\
GO:0008203, cholesterol metabolic process & -10.0957724 \\
GO:0010035, response to inorganic substance & -9.58364991 \\
\end{tabular}

there are still a lot to explore. Here, this study is designed to understand the mechanism profoundly through network pharmacology as well as molecular docking technology. Same as other network pharmacology analyses [37], taking the intersection of targets about DFD and VA in this study not only follows the operating processes of network pharmacology but also makes the result of virtual screening more reliable [61]. Undoubtedly, some targets that involve either DFD or VA might be ignored and missed, which is a common and inevitable issue in network pharmacology. And for this reason, in the collection of components and targets, despite DFD and VA, we tried our best to reduce bias by searching as much more databases as we can. Finally, so many components are boiled together, and more researches 
TABLE 4: The related information of components docked with key targets.

\begin{tabular}{|c|c|c|c|c|}
\hline Gene & Component & $\begin{array}{l}\text { PubChem } \\
\text { CLD }\end{array}$ & Origin & $\begin{array}{l}\text { Binding energy } \\
(\mathrm{kcal} / \mathrm{mol})\end{array}$ \\
\hline $\mathrm{KCNH} 2$ & Jujubogenin & 15515703 & Semen & -4.85 \\
\hline $\mathrm{KCNH} 2$ & Acacic acid lactone & 6712546 & Silktree Albizia Bark & -4.83 \\
\hline $\mathrm{KCNH} 2$ & Stepharine & 98455 & Jujubae Fructus & -4.52 \\
\hline $\mathrm{KCNH} 2$ & N-Methylasimilobine & 197017 & Semen & -4.29 \\
\hline KCNH2 & Asimilobine & 160875 & Jujubae Fructus & -4.2 \\
\hline $\mathrm{KCNH} 2$ & Cadaverine & 23335 & Semen & -4.16 \\
\hline $\mathrm{KCNH} 2$ & Nuciferine & 10146 & Jujubae Fructus & -4.07 \\
\hline $\mathrm{KCNH} 2$ & 7-Acetoxy-2-methylisoflavone & 268208 & Licorice & -4.02 \\
\hline KCNH2 & (S)-Coclaurine & 160487 & Semen; Jujubae Fructus & -3.42 \\
\hline $\mathrm{KCNH} 2$ & Juzirine & 3085285 & Semen & -3.02 \\
\hline $\mathrm{KCNH} 2$ & $\begin{array}{c}\text { (2S)-6-(2,4-Dihydroxyphenyl)-2-(2-hydroxypropan-2-yl)- } \\
\text { 4-methoxy- } \\
\text { 2,3-dihydrofuro[3,2-g]chromen-7-one }\end{array}$ & 637112 & Licorice & -2.96 \\
\hline $\mathrm{KCNH} 2$ & Zizyphusine & 102063083 & Semen & -2.8 \\
\hline $\mathrm{KCNH} 2$ & Senkyunone & 91726743 & Chuanxiong Rhizoma & -2.59 \\
\hline $\mathrm{KCNH} 2$ & 25-Hydroxy-3-epidehydrotumulosic acid & 10368709 & Poria cocos (Schw.) & -2.09 \\
\hline $\mathrm{KCNH} 2$ & Ethyl pentadecanoate & 38762 & Chuanxiong Rhizoma & -0.61 \\
\hline $\mathrm{KCNH} 2$ & AP1 & 21119850 & Silktree Albizia Bark & 1.45 \\
\hline TNNT2 & Kanzonols W & 15380912 & Licorice & -4.62 \\
\hline TNNT2 & Glabridin & 124052 & Licorice & -4.02 \\
\hline TNNT2 & Senkyunolide G & 5321250 & Chuanxiong Rhizoma & -3.23 \\
\hline TNNT2 & Odoratin & 13965473 & Licorice & -3.05 \\
\hline CALM1 & DFV & 114829 & Licorice & -4.21 \\
\hline CALM1 & Licochalcone B & 5318999 & Licorice & -2.66 \\
\hline SCN5A & Senkyunone & 91726743 & Chuanxiong Rhizoma & -1.59 \\
\hline
\end{tabular}

The binding energy refers to the strength of the binding between the receptor and the ligand; the lower the binding energy, the more stable the docking module.

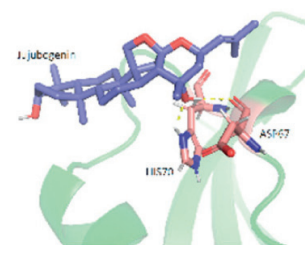

(a)

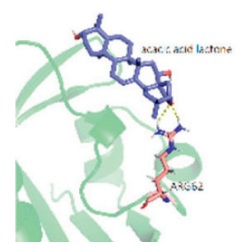

(b)

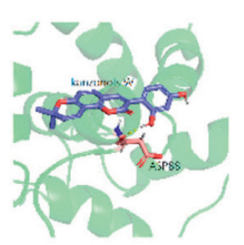

(c)

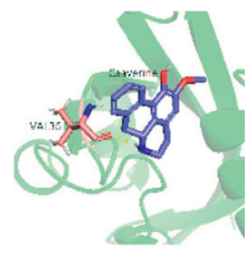

(h)

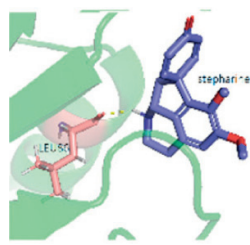

(d)

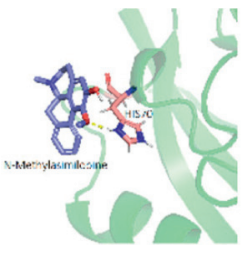

(e)

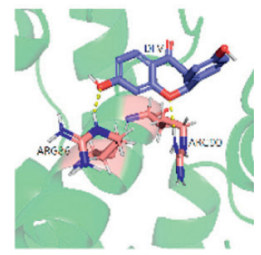

(f)

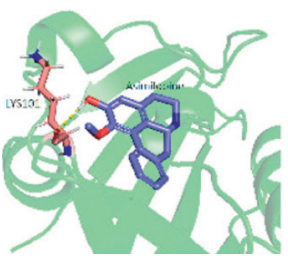

(g)

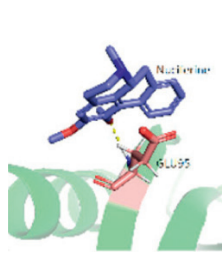

(i)

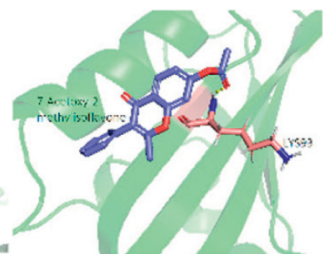

(j)

Figure 6: The top 10 binding energy docking modules.

are needed to detect whether there are some new formed compounds.

\section{Conclusion}

As mentioned above, DFD could be employed for VA through mechanisms, including complex interactions between related components and targets, as predicted by network pharmacology and molecular docking. This work confirmed that DFD could apply to the treatment of VA and promotes the explain of DFD for VA in the molecular mechanisms; similar results can be obtained from previous experiments of cellular electrophysiological mechanisms. The systematic analysis in this work can provide a comprehensive consideration for further studies.

\section{Abbreviations}

DFD: Dingji Fumai Decoction

VA: Ventricular arrhythmia 
TCM: Traditional Chinese medicine

GA: Gastrointestinal absorption

DL: Drug-likeness

H-C-T- Herb-compound-target-disease

$\mathrm{D}$ :

TCMSP: Traditional Chinese Medicine Systems Pharmacology database and analysis platform

ETCM: Encyclopedia of Traditional Chinese Medicine

ADME: Absorption, distribution, metabolism, and excretion

PPI: Protein-protein interaction

GO: $\quad$ Gene ontology

KEGG: Kyoto encyclopedia of genes and genomes

$\mathrm{KCNH} 2$ : Potassium voltage-gated channel subfamily $\mathrm{H}$ member 2

SCN5A: Sodium voltage-gated channel alpha subunit 5

TNNT2: Troponin T2-cardiac type

CALM1: Calmodulin 1

HCM: Hypertrophic cardiomyopathy.

\section{Data Availability}

All the data generated or analyzed during this study are included in this published article and its supplementary information files.

\section{Disclosure}

The manuscript's preprint was published in the following link: https://www.researchsquare.com/article/rs-117498/v1.

\section{Conflicts of Interest}

The authors declare that they have no conflicts of interest.

\section{Authors' Contributions}

Yi Liang and Bo Liang contributed equally to this work. Yi Liang, Bo Liang, and Li-Zhi Zhao conceived, designed, and planned the study. Yi Liang and Xin-Rui Wu acquired and analyzed the data. Bo Liang, Wen Chen, and Li-Zhi Zhao interpreted the results. Yi Liang and Bo Liang drafted the manuscript and Bo Liang and Li-Zhi Zhao contributed to the critical revision of the manuscript. All authors read and approved the final manuscript.

\section{Acknowledgments}

The authors thank Jing-Tong Zhao from Johns Hopkins University (Baltimore, USA) for his linguistic assistance during the preparation of this manuscript. This work was funded by the General Project of Sichuan Provincial Administration of Traditional Chinese Medicine and Research and Practice Innovation Plan for Postgraduates of Jiangsu, China.

\section{Supplementary Materials}

Table S1: comparison of Chinese medicine names and Latin names. Table S2: details of qualified compounds in various herbs. Figure S1: molecular docking modules. (Supplementary Materials)

\section{References}

[1] D. Zhao, J. Liu, M. Wang, X. Zhang, and M. Zhou, "Epidemiology of cardiovascular disease in China: current features and implications," Nature Reviews Cardiology, vol. 16, no. 4, pp. 203-212, 2019.

[2] S. M. Al-Khatib, "2017 AHA/ACC/HRS guideline for management of patients with ventricular arrhythmias and the prevention of sudden cardiac death: a report of the American College of Cardiology/American heart association task force on clinical practice guidelines and the heart Rhythm society," Journal of the American College of Cardiology, vol. 72, no. 14, pp. e91-e220, 2018.

[3] E. M. Cherry, F. H. Fenton, and R. F. Gilmour Jr., "Mechanisms of ventricular arrhythmias: a dynamical systems-based perspective," American Journal of Physiology-Heart and Circulatory Physiology, vol. 302, no. 12, pp. H2451-H2463, 2012.

[4] S. V. Pandit and J. Jalife, "Rotors and the dynamics of cardiac fibrillation," Circulation Research, vol. 112, no. 5, pp. 849-862, 2013.

[5] Y. Tsuji, "Electrical storm: recent pathophysiological insights and therapeutic consequences," Basic Research in Cardiology, vol. 108, no. 2, p. 336, 2013.

[6] P.-P. Hao, F. Jiang, Y.-G. Chen et al., "Traditional Chinese medication for cardiovascular disease," Nature Reviews Cardiology, vol. 12, no. 2, pp. 115-122, 2015.

[7] P. Hao, F. Jiang, J. Cheng, L. Ma, Y. Zhang, and Y. Zhao, "Traditional Chinese medicine for cardiovascular disease," Journal of the American College of Cardiology, vol. 69, no. 24, pp. 2952-2966, 2017.

[8] N. H. Mashour, G. I. Lin, and W. H. Frishman, "Herbal medicine for the treatment of cardiovascular disease," Archives of Internal Medicine, vol. 158, no. 20, pp. 2225-2234, 1998.

[9] X. Wang, D. Hu, S. Dang et al., "Effects of traditional Chinese medicine Shensong Yangxin capsules on heart Rhythm and function in congestive heart failure patients with frequent ventricular premature complexes," Chinese Medical Journal, vol. 130, no. 14, pp. 1639-1647, 2017.

[10] T. Wang, "A network pharmacology study of the multi-targeting profile of an antiarrhythmic Chinese medicine xin su ning," Frontiers in Pharmacology, vol. 10, p. 1138, 2019.

[11] M. Bi, S. Tong, Z. Zhang et al., "Changes in cerebral glucose metabolism in patients with mild-to-moderate Alzheimer's disease: a pilot study with the Chinese herbal medicine fuzhisan," Neuroscience Letters, vol. 501, no. 1, pp. 35-40, 2011.

[12] Y. Chen, G. Cai, X. Sun, and X. Chen, "Treatment of chronic kidney disease using a traditional Chinese medicine,Flos Abelmoschus manihot(Linnaeus) Medicus (Malvaceae)," Clinical and Experimental Pharmacology and Physiology, vol. 43, no. 2, pp. 145-148, 2016.

[13] K. Iwasaki, T. Satoh-Nakagawa, M. Maruyama et al., "A randomized, observer-blind, controlled trial of the traditional Chinese medicine Yi-Gan San for improvement of behavioral and psychological symptoms and activities of daily living in dementia patients," The Journal of Clinical Psychiatry, vol. 66, no. 2, pp. 248-252, 2005.

[14] S. Li, J. Zhao, J. Liu et al., "Prospective randomized controlled study of a Chinese herbal medicine compound Tangzu Yuyang Ointment for chronic diabetic foot ulcers: a 
preliminary report," Journal of Ethnopharmacology, vol. 133, no. 2, pp. 543-550, 2011.

[15] M. Maruyama, N. Tomita, K. Iwasaki et al., "Benefits of combining donepezil plus traditional Japanese herbal medicine on cognition and brain perfusion in Alzheimer's disease: a 12-week observer-blind, donepezil monotherapy controlled trial," Journal of the American Geriatrics Society, vol. 54, no. 5, pp. 869-871, 2006.

[16] Y. Matsuda, T. Kishi, H. Shibayama, and N. Iwata, "Yokukansan in the treatment of behavioral and psychological symptoms of dementia: a systematic review and meta-analysis of randomized controlled trials," Human Psychopharmacology: Clinical and Experimental, vol. 28, no. 1, pp. 80-86, 2013.

[17] F. J. Siddiqui, N. Venketasubramanian, E. S.-Y. Chan, and C. Chen, "Efficacy and safety of MLC601 (NeuroAiD), a traditional Chinese medicine, in poststroke recovery: a systematic review," Cerebrovascular Diseases, vol. 35, no. s1, pp. 8-17, 2013.

[18] X.-L. Tong, F.-M. Lian, Q. Zhou et al., "A prospective multicenter clinical trial of Chinese herbal formula JZQG (jiangzhuoqinggan) for hypertension," The American Journal of Chinese Medicine, vol. 41, no. 1, pp. 33-42, 2013.

[19] G. Wang, L Wang, Z. Y Xiong, B Mao, and T. Q Li, "Compound salvia pellet, a traditional Chinese medicine, for the treatment of chronic stable angina pectoris compared with nitrates: a meta-analysis," Medical Science Monitor: International Medical Journal of Experimental and Clinical Research, vol. 12, no. 1, pp. Sr1-7, 2006.

[20] Y. Wang, M Li, Y Liang et al., "Chinese herbal medicine for the treatment of depression: applications, efficacies and mechanisms," Current Pharmaceutical Design, vol. 23, no. 34, pp. 5180-5190, 2017.

[21] Y. Wang, Q Wang, C Li et al., "A review of Chinese herbal medicine for the treatment of chronic heart failure," Current Pharmaceutical Design, vol. 23, no. 34, pp. 5115-5124, 2017.

[22] H. M. Zhu, L. Qin, P. Garnero et al., "The first multicenter and randomized clinical trial of herbal Fufang for treatment of postmenopausal osteoporosis," Osteoporosis International, vol. 23, no. 4, pp. 1317-1327, 2012.

[23] X. Wang, Y. Wang, X. Y. Feng et al., "Systematic review and meta-analysis of randomized controlled trials on Wenxin keli," Drug Design, Development and Therapy, vol. 10, pp. 3725-3736, 2016.

[24] B. Liang, "Chinese herbal medicine dingji Fumai decoction for ventricular premature contraction: a real-world trial," BioMed Research International, vol. 2020, Article ID 5358467, 7 pages, 2020.

[25] B. Liang, "Antiarrhythmic mechanisms of Chinese herbal medicine dingji Fumai decoction," Evidence-Based Complementary and Alternative Medicine, vol. 2020, Article ID 9185707, 9 pages, 2020.

[26] G. B. Zhang, "Network pharmacology: a new approach for Chinese herbal medicine research," Evidence-Based Complementary and Alternative Medicine, vol. 2013, Article ID 621423, 9 pages, 2013.

[27] J. Ru, "TCMSP: a database of systems pharmacology for drug discovery from herbal medicines," Journal of Cheminformatics, vol. 6, p. 13, 2014.

[28] H.-Y. Xu, Y.-Q. Zhang, Z.-M. Liu et al., "ETCM: an encyclopaedia of traditional Chinese medicine," Nucleic Acids Research, vol. 47, no. D1, pp. D976-d982, 2019.

[29] S. Kim, P. A. Thiessen, E. E. Bolton et al., "PubChem substance and compound databases," Nucleic Acids Research, vol. 44, no. D1, pp. D1202-D1213, 2016.
[30] K. Kesarwani, R. Gupta, and A. Mukerjee, "Bioavailability enhancers of herbal origin: an overview," Asian Pacific Journal of Tropical Biomedicine, vol. 3, no. 4, pp. 253-266, 2013.

[31] A. Daina, O. Michielin, and V. Zoete, "SwissADME: a free web tool to evaluate pharmacokinetics, drug-likeness and medicinal chemistry friendliness of small molecules," Scientific Reports, vol. 7, Article ID 42717, 2017.

[32] D. Newby, A. A. Freitas, and T. Ghafourian, "Decision trees to characterise the roles of permeability and solubility on the prediction of oral absorption," European Journal of Medicinal Chemistry, vol. 90, pp. 751-765, 2015.

[33] M. Hay, D. W. Thomas, J. L. Craighead, C. Economides, and J. Rosenthal, "Clinical development success rates for investigational drugs," Nature Biotechnology, vol. 32, no. 1, pp. $40-51,2014$.

[34] A. Daina, O. Michielin, and V. Zoete, "SwissTargetPrediction: updated data and new features for efficient prediction of protein targets of small molecules," Nucleic Acids Research, vol. 47, no. W1, pp. W357-w364, 2019.

[35] J. S. Amberger and A. Hamosh, "Searching online mendelian inheritance in man (OMIM): a knowledgebase of human genes and genetic phenotypes," Current Protocols in Bioinformatics, vol. 58, pp. 1.2.1-1.2.12, 2017.

[36] D. S. Wishart, Y. D. Feunang, A. C. Guo et al., "DrugBank 5.0: a major update to the DrugBank database for 2018," Nucleic Acids Research, vol. 46, no. D1, pp. D1074-d1082, 2018.

[37] B. Liang, X.-X. Zhang, and N. Gu, "Virtual screening and network pharmacology-based synergistic mechanism identification of multiple components contained in Guanxin V against coronary artery disease," BMC Complementary Medicine and Therapies, vol. 20, no. 1, p. 345, 2020.

[38] D. Szklarczyk, A. Franceschini, S. Wyder et al., "STRING v10: protein-protein interaction networks, integrated over the tree of life," Nucleic Acids Research, vol. 43, no. D1, pp. D447-D452, 2015.

[39] P. Shannon, "Cytoscape: a software environment for integrated models of biomolecular interaction networks," Genome Research, vol. 13, no. 11, pp. 2498-2504, 2003.

[40] Y. Tang, M. Li, J. Wang, Y. Pan, and F.-X. Wu, "CytoNCA: a cytoscape plugin for centrality analysis and evaluation of protein interaction networks," Biosystems, vol. 127, pp. 67-72, 2015.

[41] G. Stelzer, "VarElect: the phenotype-based variation prioritizer of the GeneCards Suite," BMC Genomics, vol. 17, no. Suppl 2, p. 444, 2016.

[42] Y. Zhou, "Metascape provides a biologist-oriented resource for the analysis of systems-level datasets," Nature Communications, vol. 10, no. 1, p. 1523, 2019.

[43] D. S. Goodsell, C. Zardecki, L. Di Costanzo et al., "RCSB Protein Data Bank: enabling biomedical research and drug discovery," Protein Science, vol. 29, no. 1, pp. 52-65, 2020.

[44] R. E. Rigsby and A. B. Parker, "Using the PyMOL application to reinforce visual understanding of protein structure," Biochemistry and Molecular Biology Education, vol. 44, no. 5, pp. $433-437,2016$.

[45] G. M. Morris, R. Huey, and A. J. Olson, "Using AutoDock for ligand-receptor docking," Current Protocols in Bioinformatics, vol. 24, 2008.

[46] N. Ge, M. Liu, J. Krawczyk et al., "Generation and characterization of an induced pluripotent stem cell (iPSC) line (NUIGi003-A) from a long QT syndrome type 2 (LQT2) patient harbouring the KCNH2 c.2464G>A pathogenic variant," Stem Cell Research, vol. 49, p. 101997, 2020. 
[47] R. Walsh, "Enhancing rare variant interpretation in inherited arrhythmias through quantitative analysis of consortium disease cohorts and population controls," Genetics in medicine: official journal of the American College of Medical Genetic, vol. 23, no. 1, pp. 47-58, 2021.

[48] S. Ballouz, "Co-expression of calcium and hERG potassium channels reduces the incidence of proarrhythmic events," Cardiovascular Research, 2020.

[49] J. E. Ezekian, "Variant R94C in TNNT2-encoded Troponin T predisposes to pediatric restrictive cardiomyopathy and sudden death through impaired thin filament relaxation resulting in myocardial diastolic dysfunction," Journal of the American Heart Association, vol. 9, no. 5, Article ID e015111, 2020.

[50] Y. Wu, Z. Zuo, M. Chen et al., "The developmental effects of low-level procymidone towards zebrafish embryos and involved mechanism," Chemosphere, vol. 193, pp. 928-935, 2018.

[51] R. Coppini, C. Y. Ho, E. Ashley et al., "Clinical phenotype and outcome of hypertrophic cardiomyopathy associated with thin-filament gene mutations," Journal of the American College of Cardiology, vol. 64, no. 24, pp. 2589-2600, 2014.

[52] G. J. Kang, "MIR448 antagomir reduces arrhythmic risk after myocardial infarction by upregulating the cardiac sodium channel," JCI Insight, vol. 23, no. 5, 2020.

[53] J. Zakrzewska-Koperska, "A combination of quinidine/ mexiletine reduces arrhythmia in dilated cardiomyopathy in two patients with R814W SCN5A mutation," ESC Heart Failure, vol. 7, no. 6, pp. 4326-4335, 2020.

[54] L. M. Wren, "Genetic mosaicism in calmodulinopathy," Circulation: Genomic and Precision Medicine, vol. 12, no. 9, pp. 375-385, 2019.

[55] W. C. Tsai, "Complex arrhythmia syndrome in a knock-in mouse model carrier of the N98S Calm1 mutation," Circulation, vol. 142, no. 20, pp. 1937-1955, 2020.

[56] L. Crotti, C. Spazzolini, D. J. Tester et al., "Calmodulin mutations and life-threatening cardiac arrhythmias: insights from the International Calmodulinopathy Registry," European Heart Journal, vol. 40, no. 35, pp. 2964-2975, 2019.

[57] D. E. Arking, S. L Pulit, L Crotti et al., "Genetic association study of QT interval highlights role for calcium signaling pathways in myocardial repolarization," Nature Genetics, vol. 46, no. 8, pp. 826-836, 2014.

[58] L. A. Venetucci, A. W Trafford, S. C O’Neill, and D. A Eisner, "The sarcoplasmic reticulum and arrhythmogenic calcium release," Cardiovascular Research, vol. 77, no. 2, pp. 285-292, 2008.

[59] D. Lang, K. Holzem, C. Kang et al., "Arrhythmogenic remodeling of $\beta 2$ versus $\beta 1$ adrenergic signaling in the human failing heart," Circulation: Arrhythmia and Electrophysiology, vol. 8, no. 2, pp. 409-419, 2015.

[60] P. Zhang, C. M. Kofron, and U. Mende, "Heterotrimeric G protein-mediated signaling and its non-canonical regulation in the heart," Life Sciences, vol. 129, pp. 35-41, 2015.

[61] S. Li and B. Zhang, "Traditional Chinese medicine network pharmacology: theory, methodology and application," Chinese Journal of Natural Medicines, vol. 11, no. 2, pp. 110-120, 2013. 